\title{
Congo Red (CRD) Test in Prediction of Preeclampsia: An Innovative Mobile Health Solution
}

Preeclampsia (PE) is a life-threatening complicated pregnancy disorder characterized by new onset of hypertension (systolic and diastolic blood pressure of $\geq 140$ and $90 \mathrm{~mm} \mathrm{Hg}$ respectively), proteinuria (on dipstick $\geq 2+$ ), edema and thrombocytopenia (platelet count $<100,000 / \mu \mathrm{L}$ ) after 20 weeks of gestation. If left untreated, can be fatal for both the mother and child. PE has been associated with maternal organ dysfunction-includes renal insufficiency, inappropriate liver function, fetal growth retardation, neurological and hematological complications. ${ }^{1}$ It affects $2-8 \%$ of all pregnancies leading to prenatal morbidity and mortality worldwide. The epidemiology of preeclampsia is reported to be $8-10 \%$ of the pregnancies in India. ${ }^{2}$ The prevalence of the disease might vary in different populations and different ethnic groups. The pathogenesis of PE remains unclear but placenta plays an important role in development of the disease by mediating the poor placental development, endothelial dysfunction, trophoblast damage and aberrant angiogenesis. In preeclampsia, placenta becomes hypoxic which might trigger oxidative stress and increased placental apoptosis leading to

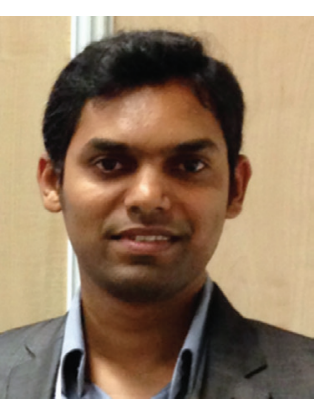
defective endothelial dysfunction, uteroplacental circulation and worsens the placental perfusions. ${ }^{3}$ Therefore, failure of endothelial vascular development causes high blood pressure, increased vascular permeability and proteinuria. The clinical features of preeclampsia are unified and it targets the organs, which may cause seizures and stroke (brain), HELLP (hemolysis elevated liver enzymes, low platelet count (liver)) and proteinuria, glomerular endotheliosis (kidney). Several angiogenic factors such as soluble FMS-like tyrosine kinase -1(sFlt1), vascular endothelial growth factor (VEGF-1), soluble Endoglin (sEndoglin), placental growth factor (PIGF) are thought to be responsible for the regulation of placental vascular development. The only definitive treatment for this condition is early delivery of the fetus and the placenta. There are several risk factors causing the disease that include previous history of pregnancy and certain medical conditions such as gestational diabetes, type I diabetes, obesity, kidney diseases, thrombophilias, chronic high blood pressure, urinary tract infections and periodontal disease during pregnancy increase the risk of preeclampsia. The early and late pregnancy between age less than 20 and greater than 35 weeks, women who are carrying twins or more, prolonged interval pregnancies and women who develop gestational diabetes are at greater risk of developing PE. ${ }^{4}$ Therefore, prediction or detection of PE earlier is more important and biomarker plays an important role in prediction of PE earlier. A wide range of potential, novel biomarkers are being investigated and angiogenic factors are suggested for prediction of PE. Maternal plasma levels of these factors can indentify most patients in developing early preeclampsia correlated with disease severity and have prognostic value for maternal and/or perinatal complications. ${ }^{5}$ The availability of one or more reliable biomarkers helps to determine clinical responsiveness to diagnosis. Recent studies have reported that preeclampsia is a protein conformational disorder and associated with presence of amyloid proteins. Similar to amyloid proteins in Alzheimer's disease, Parkinson's disease, Huntington's disease, PE showed affinity towards Congo red dye. ${ }^{6}$ It is proposed that amyloid proteins have a propensity to bind to Congo red dye excreted in the urine of preeclampsia women, well before the onset of clinical symptoms and activation of unfolded response pathway in the placenta is different between early-onset and late-onset PE. The maternal factor that leads to urinary misfolded protein appears to be shared by both early-onset and late-onset preeclampsia and thus represents a core pathophysiological event. These misfolded proteins accumulate in the urine, serum and the placenta of women with PE. A study by Buhimschi et al. demonstrated that Congo red test is useful for early diagnosis of preeclampsia and can be detected by a simple dot test technique using Congo red dye that binds with specific affinity to the $\beta$-sheet of amyloid fibrils of misfolded proteins. ${ }^{7}$ A study by Sailakshmi et al. confirmed the presence of urinary congophilia protein, and its usefulness in early prediction of preeclampsia using CRD test. This test also showed the presence of congophilia in PE women by 4-7 weeks prior to the onset of clinical symptoms. The sensitivity and the specificity of the test was $66.18 \%$ and $96.45 \%$ respectively. The positive predictive value (PPV) and negative predictive value (NPV) were $80.36 \%$ and was $92.86 \%$ respectively. ${ }^{8}$ A study by Nagarajappa et al. has shown that urinary congophilia level was elevated in preeclamptic women compared to normotensive pregnant women in the Indian population. The level of urine congophilia varies across the clinical subgroups of preeclampsia but the average level remains significantly higher than the normotensive pregnant women. ${ }^{9}$ The CRD test is now being investigated as an innovative mobile health solution in countries with limited resources as a diagnostic and prognostic tool for preeclampsia. The CRD test was not only effective in predicting preeclampsia but also useful in differentiating between preeclampsia and other forms of hypertension, as well as early onset and late onset preeclampsia. The CRD test can be used as a rapid test in prediction of PE and also as a diagnostic marker for the early diagnosis prior to the clinical manifestation of PE in low-income settings, as it is being an inexpensive, accurate, non-invasive, rapid and easy-to-perform test, with good specificity and sensitivity.

Anbazhagan Kolandaswamy Associate Professor, Department of Molecular Medicine Central Research Lab, RajaRajeswari Medical College and Hospital Bengaluru-560074, India e-mail: anbu50@gmail.com Phone: 9066607714 


\section{References}

1. Ben WJ Mol, Claire T Roberts, Shakila Thangaratinam, Laura A Magee, Christianne JM de Groot, G Justus Hofmeyr. Preeclampsia. September 3, Lancet 2016; 387: 999-1011.

2. Arun Jeyabalan. Epidemiology of preeclampsia: Impact of obesity. Nutr Rev. 2013 Oct; 71(01): 10.1111/nure.12055.

3. Al-Jameil N, Aziz Khan F, Fareed Khan M, Tabassum H. A brief overview of preeclampsia. Journal of Clinical Medicine Research. Feb 2014;6(1):1-7.

4. Mustafa R, Ahmed S, Gupta A, Venuto RC. A comprehensive review of hypertension in pregnancy. Journal of Pregnancy. 2012:1-19.

5. Petla LT, Chikkala R, Ratnakar KS, Kodati V, Sritharan V. Biomarkers for the management of preeclampsia in pregnant women. Indian J Med Res. 2013;138:60-7.

6. Sweeney P, Park H, Baumann M, Dunlop J, Frydman J, Kopito R, McCampbell A, Leblanc G, Venkateswaran A, Nurmi A, Hodgson R. Protein misfolding in neurodegenerative diseases: implications and strategies, Transl. Neurodegener. 2017;6:1-13. https://doi.org/10.1186/s40035-017-0077-5.

7. Buhimschi IA, Nayeri UA, Zhao G, Shook LL, Pensalfini A, Funai EF, Bernstein IM, Glabe CG, Buhimschi CS. Protein misfolding, congophilia, oligomerization, and defective amyloid processing in preeclampsia. Sci Transl Med. 2014;6. https:// doi.org/10.1126/scitranslmed.3008808.

8. Sailakshmi MPA, Prabhu MR, Prabhakara S, Anbazhagan K, Rupakala BM. Congo red dot test in the early prediction and diagnosis of preeclampsia in a tertiary health care centre in India. Pregnancy Hypertension. 2021;25:225-229.

9. Nagarajappa C, Rangappa SS, Suryanarayana R, Balakrishna S. Urinary congophilia in preeclampsia: Experience from a rural tertiary-care hospital in India. Pregnancy Hypertension. 2018;13:83-86. 